\title{
Enhancement of the efficiency of PEI/liposome transfection by magnetofectins formed via electrostatic self-assembly
}

\author{
MA YongJie, WANG XiaoLiang \& GU HongChen* \\ Nano Biomedical Research Center, School of Biomedical Engineering, Shanghai Jiao Tong University, Shanghai 200030, China
}

Received October 10, 2011; accepted February 3, 2012; published online May 15, 2012

\begin{abstract}
One of the major challenges for successful gene therapy is improving the transfection efficiency of non-viral vectors. Magnetic nanoparticles (MNPs) have been developed as enhancers of non-viral vehicles. We prepared MNPs and modified them with polyethyleneimine (PEI), citric acid (CA) or carboxylmethyl-dextran (CMD). Both positively charged MNPs (MNPs@PEI) and negatively charged MNPs (MNPs@CA, MNPs@CMD) could spontaneously form transfection complexes (magnetofectins) with plasmid DNA and PEI/liposome via electrostatic self-assembly. Our results showed as-prepared magnetofectins apparently enhanced PEI/liposome transfection efficiency and/or gene expression level into COS-7 cells with reduced transfection time from $4 \mathrm{~h}$ to $15 \mathrm{~min}$ under a magnetic field in vitro. Meanwhile, the effect of magnetofection was cell line-dependant. These results suggest that charged MNPs could improve transfection efficiency for non-viral vectors by simply mixing with them and by exerting a magnetic force. Thus such MNPs provide a convenient platform for further applications of gene delivery.
\end{abstract}

gene delivery, liposome, magnetofection, magnetic nanoparticles, PEI

Citation: Ma Y J, Wang X L, Gu H C. Enhancement of the efficiency of PEI/liposome transfection by magnetofectins formed via electrostatic self-assembly. Chin Sci Bull, 2012, 57: 4005-4011, doi: 10.1007/s11434-012-5184-1

Efficient gene delivery remains a challenge for gene therapy. Non-viral vectors have the advantages of safety and flexibility over viral vectors [1,2]. However, the transfection efficiencies of non-viral gene vectors are generally lower than those of viral vectors [3]. In order to improve the transfection efficiency of non-viral vectors, a new technology termed "magnetofection" has been developed [3-5]. With this technology, the complexes of nucleic acids and their vectors are combined with MNPs to form "transfection ternary complexes" termed magnetofectins [4], which can be concentrated to the target cells by a magnetic field. The transfection efficiency of certain gene vectors can be enhanced while reducing transfection time [5].

In order to combine MNPs with non-viral vectors, MNPs were coated with PEI $[4,5]$ or cationic liposomes [6]. In fact, according to the mechanism study on magnetofection, MNPs play the role of driving vector/pDNA complexes to cell surface in magnetofection and do not directly affect the

*Corresponding author (email: hcgu@ @stu.edu.cn) endocytic uptake mechanism [4]. Magnetofection requires MNPs to have sufficient electric potentials at the surface, so it is not necessary to modify MNPs with these gene vectors according to our previous study [7]. Our experimental evidences indicate that MNPs could not enter nucleus once they were internalized by the cells. MNPs would probably have to be separated from vector/pDNA complexes after entering cytoplasm, although detailed mechanism requires further investigation. In this study, we aim to further demonstrate charged MNPs, both positively charged and negatively charged MNPs, can improve transfection efficiency for non-viral vectors by simply mixing with them via electrostatic self-assembly.

In this paper, we introduced the preparation, cytotoxicity of magnetofectins as prepared via electrostatic self-assembly, and evaluated the performance of these magnetofectins in magnetofection as follows: (1) to determine if MNPs@PEI can improve PEI reagent transfection; (2) to determine if negatively charged MNPs@CA and MNPs@CMD can improve PEI reagent transfection; (3) to determine if charged 
MNPs can improve Liposome reagent transfection; and (4) to compare the transfection effects of magnetofection with lipofection in different cell lines.

\section{Materials and methods}

\subsection{Materials and equipments}

(1) Materials. MTT solution and dimethyl sulfoxide (DMSO) were from Sigma Aldrich. PEI (25 kD) was from Sigma Aldrich. DMEM culture medium (high glucose) and fetal calf serum were obtained from Hyclone. Penicillin/streptomycin and Lipofectamine- $2000^{\mathrm{TM}}$ reagent were obtained from Invitrogen. Plasmid expressing enhanced green fluorescent protein (pEGFP-C1) was from Clontech and plasmid expressing luciferase (pGL3-control vector) was from Promega. All other reagents were obtained from Shanghai Reagent Company.

COS-7 (SV 40 transformed kidney cells of African green monkey), CHO-K1 (Chinese hamster ovary cells), PC-12 (clonal rat pheochromocytoma cells) and P815 (mouse lymphoblast-like mastocytoma cell line) cell lines were purchased from the Cell Bank of Chinese Academy of Sciences.

(2) Equipments. The equipments used in this study are transmission electron microscopy (TEM, Philips CM-120), Zetasizer 2000 (Malvern), magnetic plate (MagnetoFACTOR plate, Chemicell), Multilabel Counter (Victor-3, Perkin Elmer) and fluorescent microscope (Leica DMI 3000B).

\subsection{Methods}

(1) Preparation of charged MNPs and magnetofectins. Charged MNPs (MNPs@PEI, MNPs@CA and MNPs@CMD) were prepared according to methods described elsewhere [7]. Modified MNPs were observed by TEM and their zeta potentials were measured by a Zetasizer.

To prepare PEI-based magnetofectins, $3 \mu \mathrm{g}$ of plasmid expressing enhanced green fluorescent protein or plasmid expressing luciferase was suspended in $100 \mu \mathrm{L}$ serum-free DMEM buffer followed by adding charged MNPs according to mass ratios of MNPs to pDNA $(0.1,0.2,0.4$ or 1.0$)$ and incubated at room temperature for $10 \mathrm{~min}$. The $0.26 \mathrm{mg} / \mathrm{mL}$ PEI solution ( $1 \mu \mathrm{g}$ of DNA corresponds to $3 \mathrm{nmol}$ of phosphate, and $1 \mu \mathrm{L}$ of PEI corresponds to $6 \mathrm{nmol}$ of amine nitrogen) was added according to the ratios of amine nitrogen of PEI to phosphate of DNA $(N / P, 10,20$ or 30) and incubated at room temperature for another $15 \mathrm{~min}$.

To prepare Liposome-based magnetofectins, standard complexes of pEGFP-C1 and Lipofectamine- $2000^{\mathrm{TM}}$ reagent were prepared according to the protocol followed by mixing with charged MNPs. In liposome-based magnetofectins, the M/M of MNPs to pDNA was 0.4.

About the above magnetofection formulation, $3 \mu \mathrm{g}$ pDNA and $1.2 \mu \mathrm{g}$ MNPs were required for each well of a 6-well plate, and $0.2 \mu \mathrm{g}$ pDNA and $0.15 \mu \mathrm{g}$ MNPs were required for each well of a 96-well plate.

(2) Cell culture. COS-7, CHO-K1, PC-12 and P815 cell lines were cultured in DMEM supplemented with $10 \%$ (v/v) fetal bovine serum and $1 \%$ penicillin/streptomycin at $37^{\circ} \mathrm{C}$ in a humidified $5 \% \mathrm{CO}_{2}$ atmosphere.

(3) MTT assay. Cytotoxicity of magnetofectins was evaluated by the MTT assay. Cells were seeded in 96-well plates $\left(1 \times 10^{5}\right.$ cells/well) with $200 \mu \mathrm{L}$ DMEM in each well and incubated at $37^{\circ} \mathrm{C}$ in a humidified atmosphere $\left(5 \% \mathrm{CO}_{2}\right)$ for $24 \mathrm{~h}$. After removing culture media, a control group was treated with $100 \mu \mathrm{L} 0.9 \%$ physiological saline while the experimental groups were treated with $100 \mu \mathrm{L}$ PEI/pDNA transfection complexes $(0.2 \mu \mathrm{g}$ pDNA, $N / P=20)$ or PEI-based magnetofectins.

Magnetofectins were formed by mixing MNPs@PEI, MNPs@CA or MNPs@CMD with pEGFP-C1 $(M / M=0.2$, 0.4 or 1.0$)$ followed by mixing with PEI $(N / P=20)$. According to the transfection protocol, COS-7 cells were treated with PEI/pDNA transfection complexes for $4 \mathrm{~h}$ while they were treated with PEI-based magnetofectins for 15 min at a magnetic plate. All the cell samples were incubated again for another $24 \mathrm{~h}$ before $20 \mu \mathrm{L}$ of $5 \mathrm{mg} / \mathrm{mL}$ MTT solution in PBS was added to each well. The plates were incubated once more for $4 \mathrm{~h}$. Then, the MTT-containing media was removed and $150 \mu \mathrm{L}$ DMSO was added to dissolve the formazan crystals produced from the reduction of MTT by viable cells. The optical density was measured by Multilabel Counter at $570 \mathrm{~nm}$. The cell viability was calculated by normalizing the OD values of the experimental groups to that of the control cells.

(4) Magnetofection. In order to determine if MNPs@PEI could improve PEI reagent transfection, COS-7 cells were transfected by MNPs@PEI-based magnetofectins with pEGFP-C1 or pGL3-control vector pDNA compared with $\mathrm{PEI}$ transfection. Magnetofectins were prepared at $M / M(0.1$, $0.2,0.4$ or 1.0$)$ and $N / P(10,20$ or 30$)$ according to the methods described in Section 1.2 (1).

In order to determine if negatively charged MNPs@CA and MNPs@CMD could improve PEI reagent transfection, COS-7 cells were transfected with magnetofectins that were formed based on the complexing of MNPs@PEI, MNPs@CA, or MNPs@CMD with pGL3-control vector pDNA Magnetofectins were prepared at $M / M(0.2,0.4$ or 1.0$)$ and $N / P 20$.

In order to determine if MNPs@PEI, MNPs@CA and MNPs@CMD could improve Liposome reagent transfection, COS-7 cells were transfected by magnetofectins $(M / M=0.4)$ with pEGFP-C1 compared with Lipofectamine- $2000^{\mathrm{TM}}$ transfection.

In order to compare the transfection efficiency of magnetofection with lipofection, CHO-K1, PC-12 and P815 cell lines were transfected by MNPs@PEI $(M / M=0.4, N / P=20)$ with pEGFP-C1 compared with Lipofectamine- $2000^{\mathrm{TM}}$ transfection.

During the magnetofection process, magnetofectins were 
added to serum-free cell culture media and then the cell culture plate (6-well plate or 96-well plate) was placed on the magnetic plate $(200 \mathrm{mT})$ for $15 \mathrm{~min}$. The media was removed from each well and replaced with media supplemented with fetal calf serum. Cells were subsequently cultured for $48 \mathrm{~h}$ before harvest, and the transfection efficiency was determined by counting the percentage of EGFP positive cells via fluorescent microscope and the levels of luciferase expression were determined via the Multilabel Counter. All experiments were performed in triplicate.

\section{Results and discussions}

\subsection{Characterizations of charged MNPs}

The surface modified particles were from one single batch of native particles synthesized by the co-precipitation methods [8]. The surface modification as characterized by the change of the particles' Zeta potentials, was shown in Figure 1d. MNPs coated with CA or CMD were negatively charged and MNPs coated with PEI were positively charged, which were in accordance with the theoretical predictions. Furthermore, it was also indicated that the proposed com- ponents had been successfully coated onto the MNPs. The colloidal stability had essential effects on magnetofection efficiency. Since the modified MNPs exhibited appreciable surface charges, they were consequently well-dispersible in water (Figure 1a-c).

\subsection{MTT assays}

To test whether magnetofectins as prepared by three different approaches posed any cytotoxicity, at least in the quantity range used for transfection, we evaluated viability of COS-7 cells in the presence of these particles. The conditions of the experimental groups in the MTT assay were in accordance with the actual transfection protocols. All the magnetofectins composed of MNPs@PEI, MNPs@CA or MNPs@CMD showed lower cytotoxicity than PEI/DNA complexes (Figure 2). In the standard PEI transfection, cells would need $4 \mathrm{~h}$ of incubation in serum-free and PEIcontaining media, to achieve considerable transfection efficiency, whereas in the magnetofection groups using charged MNPs as transfection enhancers, the required time was shortened to only $15 \mathrm{~min}$. This time difference produced one of the advantages of using magnetofection for gene
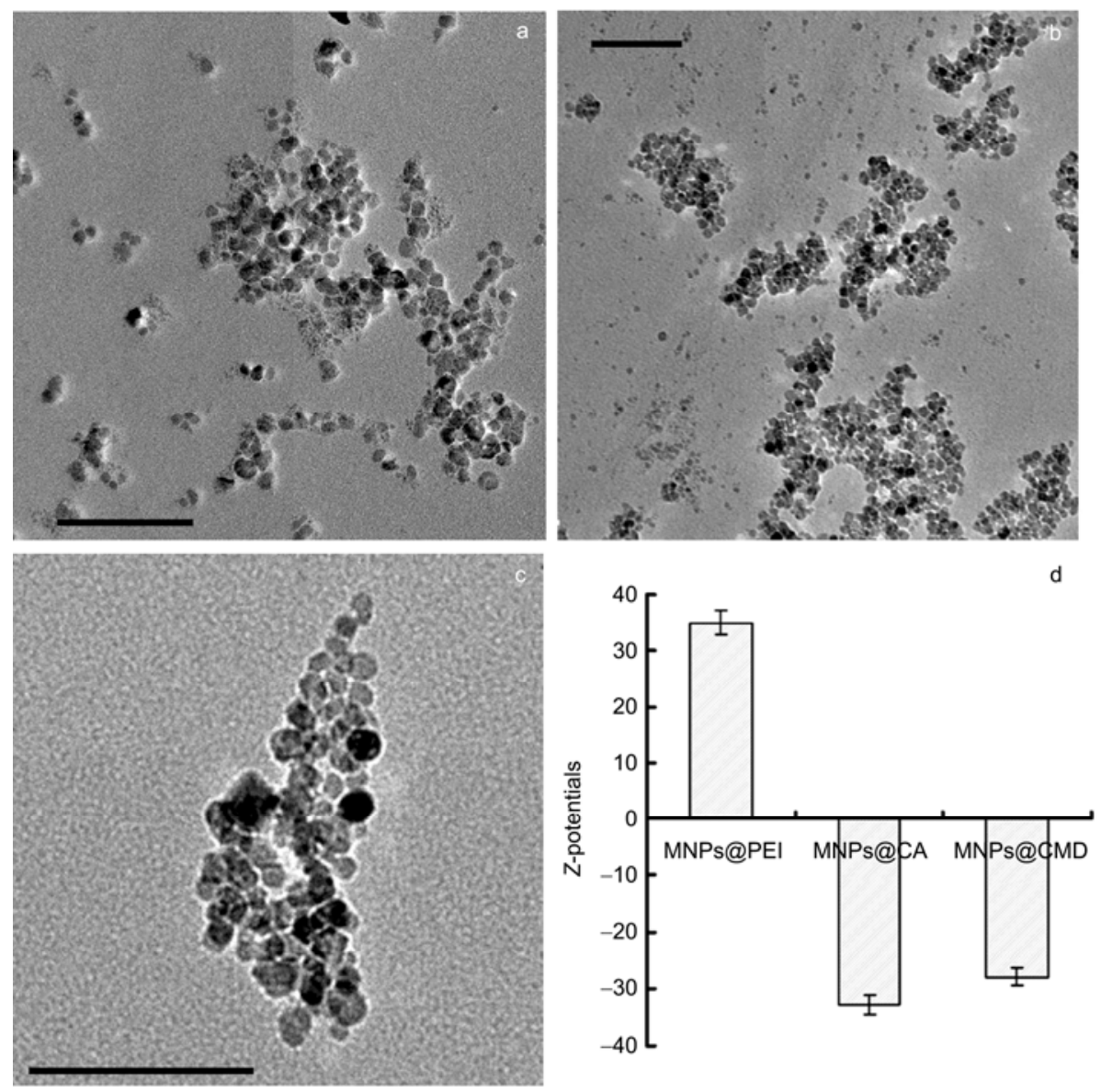

Figure 1 Characterizations of charged MNPs. a, TEM images of MNPs@PEI; b, MNPs@CA; c, MNPs@CMD; d, zeta potentials of modified MNPs (mean $\pm \mathrm{SD}, n=3$ ). Scale bars are $100 \mathrm{~nm}$. 
delivery. Moreover, this difference could also probably explain why the magnetofection did less harm to the cells. With shorter exposure, the cells were likely to be less affected. Based on published literature $[9,10]$ and our own data (Figure 2), the cytotoxicity was mainly due to PEI. It appeared that the different mass ratios of MNPs to pDNA (from 0.2 to 1.0) almost had no influence on the cytotoxicity.

\subsection{MNPs@PEI enhanced PEI gene transfection}

COS-7 cells were transfected by MNPs@PEI-based magnetofectins for $15 \mathrm{~min}$ on top of the magnetic plate, compared with PEI standard transfection that required $4 \mathrm{~h}$ incubation. The transfection enhancement, as determind by the luciferase expression efficiencies for pGL3-control vector, and by the percentage of GFP-positive cells for pEGFP-C1 is shown in Figure 3. MNPs@ PEI apparently improved PEI transfection rate and improved transgene expression level with significantly reduced transfection time.

As Figure 2 showed, the reduced transfection time could also reduce the cytotoxicity. On the other hand, the magnetic field could drive the vectors towards the target cells. In this manner, the transfection complexes were concentrated on the cells surface within a few minutes so that most of the cells got in contact with a significant vector dose $[11,12]$. According to our stability studies on the magnetofectins, different $N / P$ ratios $(10,20$ or 30$)$ did not influence their stability and magnetofectins could be sedimented by a magnetic force (Figure 3), so that all the magnetofectins formed at different $N / P$ acted as enhancers in magnetofection. Based on the cytotoxic evaluation of magnetofectins, different $M / M$ ratios did not apparently influence their cytotoxicity (Figure 2). This might be one of the reasons for the similar transfection results between magnetofectins formed at different $M / M$ ratios. Compared with the effects on transfection efficiency affected by $M / M$ ratios, we consider more effects affected by different $N / P$ ratios. However, the relationship between them needs to be further elucidated.

\subsection{Charged MNPs enhanced PEI and liposome- mediated gene transfections}

If charged MNPs have sufficient electric potentials at their surfaces, either negative charges or positive charges, they could form magnetofectins via electrostatic self-assembly with pDNA and PEI. Positively charged MNPs could form complexes with negative charged pDNA, while negatively charged MNPs could form complexes with positively charged PEI. Although the transfection conditions in COS-7 cells need to be further optimized, magnetofectins formed at $N / P$ 20 showed better performance in magnetofection (Figures 4 and 5). We chose the condition of N/P 20 to determine the performances of three kinds of charged MNPs in magnetofection.

Charged MNPs including MNPs@PEI, MNPs@CA and MNPs@CMD apparently improved the transgene expression levels of pGL3-control vector to COS-7 cells compared with PEI standard transfection (Figure 4). From the results shown in Figure 4, we might infer that the $M(\mathrm{MNPs}) / M$ (pDNA) ratios do not apparently affect the transfection efficiency, at least in the range we tested.

We prepared the transfection complexes of pDNA/liposome according to the protocol followed by the addition of the charged MNPs. The different magnetofectins were used to transfect COS-7 cells with pEGFP-C1. The difference in the level of green fluorescence was obvious: the percentage of GFP-positive cells transfected with MNPs@CA- or MNPs@CMD-based magnetofectins for $15 \mathrm{~min}$ was much more than that transfected by Liposome- $2000^{\mathrm{TM}}$ for $15 \mathrm{~min}$ or $4 \mathrm{~h}$ (Figure 5). With the same transfection time period, transfection rate with MNPs@PEI-based magnetofection was almost the same as the one with Lipofectamine transfection

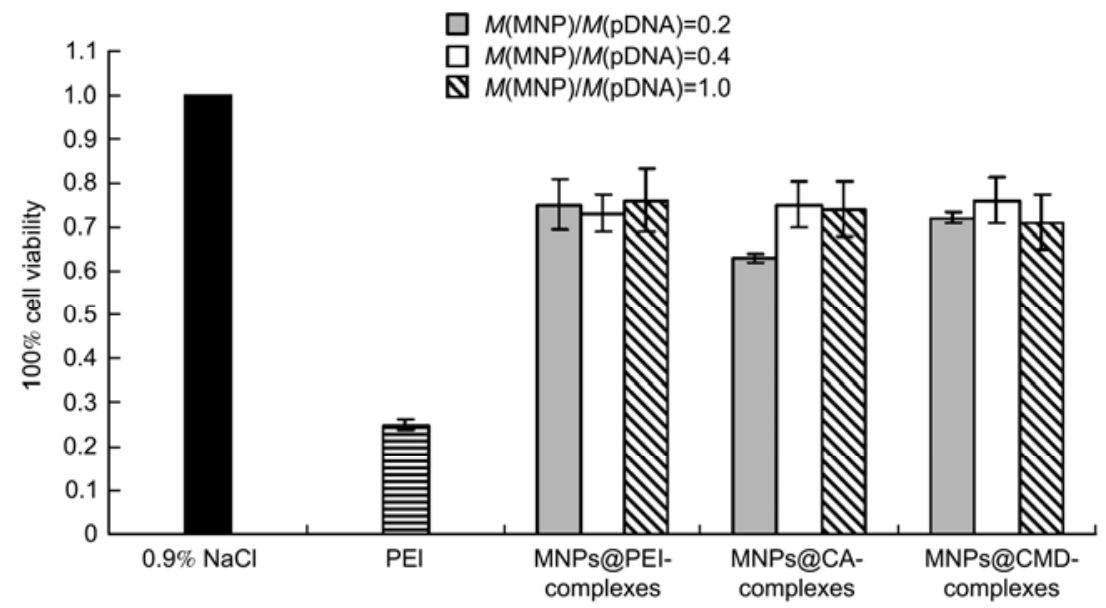

Figure 2 MTT assay to determine cytotoxicity of the transfection complexes. COS-7 cells were incubated with magnetofectins for 15 min or PEI-DNA compounds for $4 \mathrm{~h}$, and then processed for MTT assay. In the magnetofectins, the mass ratios of MNPs to pDNA are $0.2,0.4$ or 1.0 . Viability of cells was shown compared to physiological saline-treated cells (control) (mean $\pm \mathrm{SD}, n=3$ ). 
a
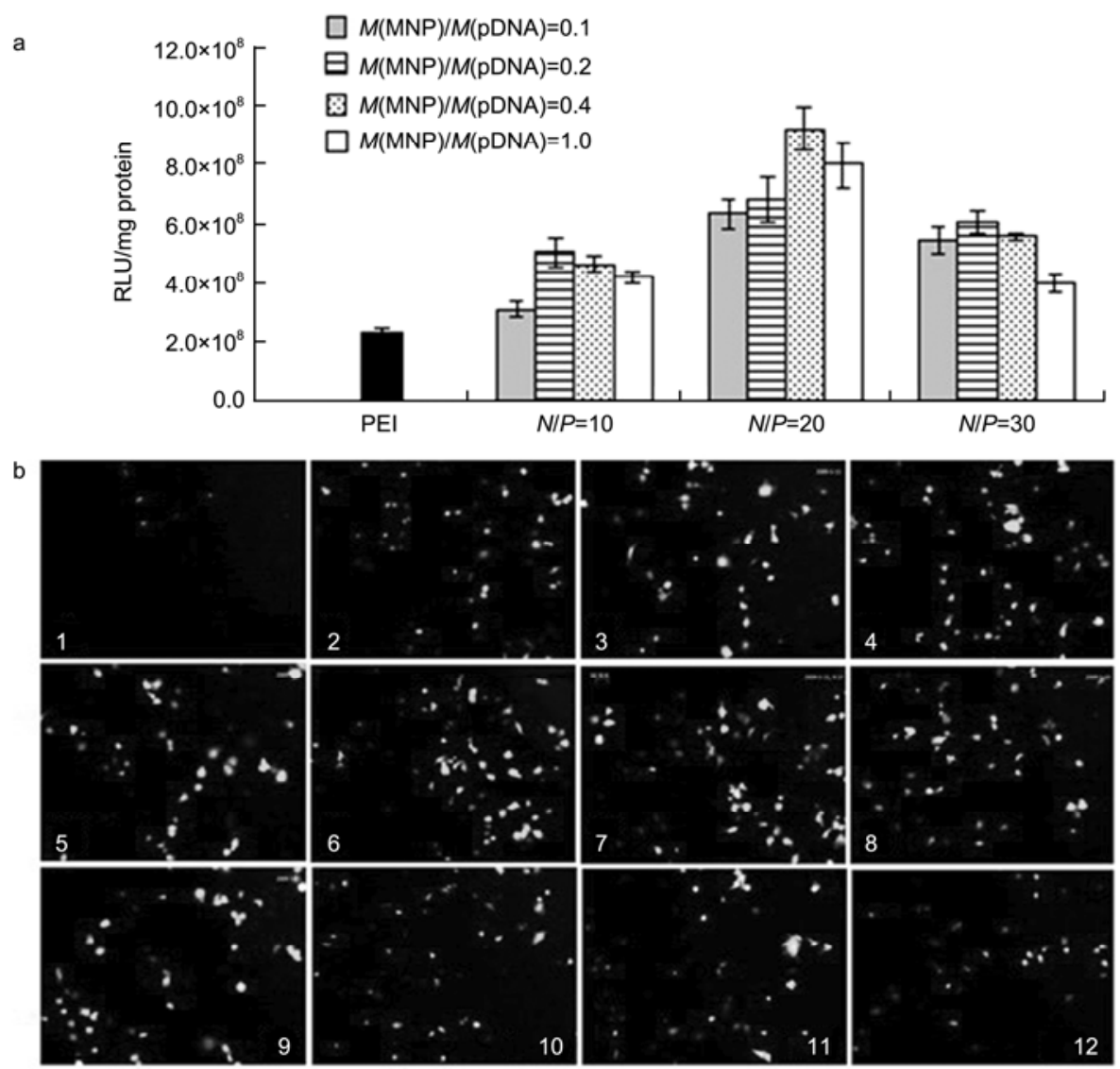

\begin{tabular}{|l|l|l|l|}
\hline PEI transfection & $N / P=10, M / M=0.2$ & $N / P=10, M / M=0.4$ & $N / P=10, M / M=1.0$ \\
\hline$N I P=20, M / M=0.1$ & $N / P=20, M / M=0.2$ & $N / P=20, M / M=0.4$ & $N / P=20, M / M=1.0$ \\
\hline$N / P=30, M / M=0.1$ & $N / P=30, M / M=0.2$ & $N / P=30, M / M=0.4$ & $N / P=30, M / M=1.0$ \\
\hline
\end{tabular}

Figure 3 Quantitative and qualitative analysis of pGL3-control vector and pEGFP-C1 transfection to COS-7 cells with multilabel counter (a, mean \pm SD, $n=3)$; b, fluorescent microscope. Magnetofectins formed by mixing MNPs@PEI with pDNA at different $M / M(0.1,0.2,0.4$ or 1.0$)$ and followed by mixing PEI with pDNA at different N/P (10, 20 or 30). PEI transfection was used as a control group. The descriptions of $1-12$ in b are listed in the lower table.

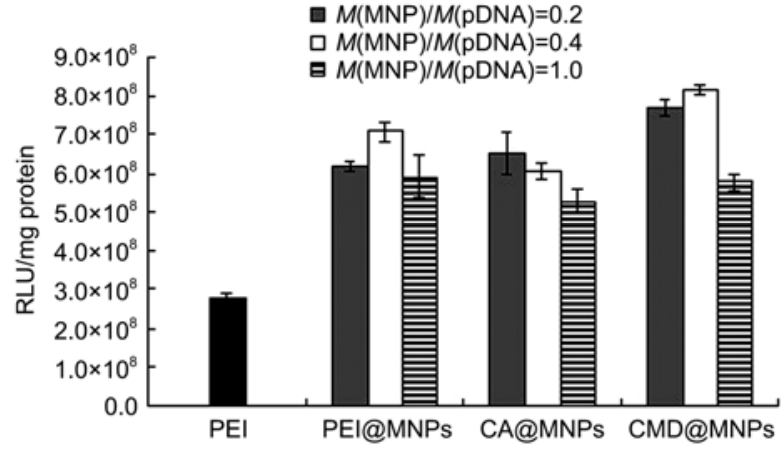

Figure 4 Charged MNPs enhanced transgene expression levels in COS-7 cells compared with PEI standard transfection $(N / P=20$, mean $\pm \mathrm{SD}, n=3)$ Relative luciferase activity (RLU/mg of total protein) was used to compare the transfection efficiency.

(Figure 5b,c). Despite the different surface charges on MNPs, we prepared liposome-based magnetofectins at the same mass ratios and N/P ratios. Liposome is widely used for transfection, but what makes the transfection inconven- ient is that the culture media usually need be changed after $4 \mathrm{~h}$ incubation. Furthermore, it is difficult for liposome to be used in vivo. In our experiments, we tested liposome-based transfection with $15 \mathrm{~min}$ incubation as well, and found this shortened incubation did not apparently alter transfection efficiency. Surprisingly, with the addition of MNPs@ PEI, MNPs@CA and MNPs@CMD into liposome-based transfection system (Figure $5 \mathrm{c}-\mathrm{e}$ ) with 15 min incubation, the transfection efficiency as indicated by percentage of GFP + cells, increased significantly (Figure 5c-e vs. a,b). It seemed that MNPs@CA, MNPs@CMD could enhance liposome transfection effects more than MNPs@PEI (Figure $5 \mathrm{~d}$,e vs. c). The above results might suggest liposome transfection could be more convenient and highly efficient by adding magnetic enhancers and might mean the possibility of remotely controlled vector targeting in vivo. This fact also implies that the transfection efficiency might be improved if MNPs modified with enough charge on the surface are added to other non-viral/pDNA complexes at certain $M / M$ and $N / P$ ratios, and a magnetic force is exerted. 

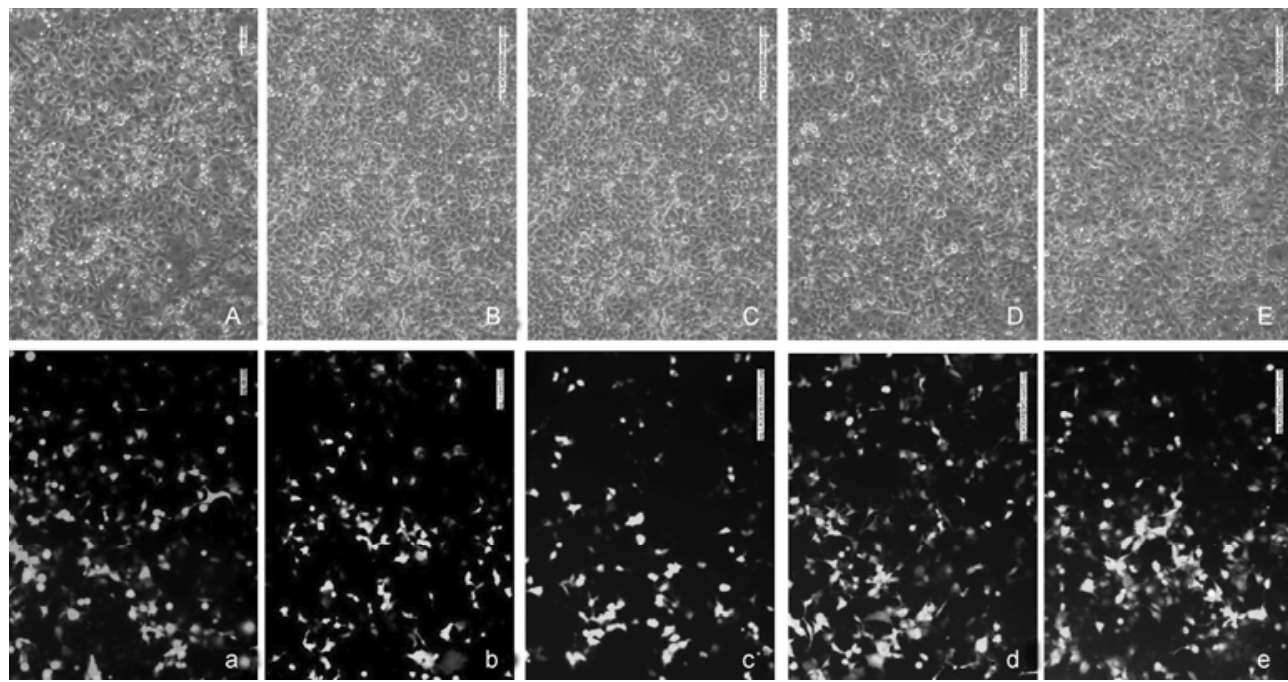

Figure 5 Charged MNPs enhanced GFP-positive cell-percentages of COS-7 cells compared with liposome transfection. a, Lipofectamine-2000 ${ }^{\mathrm{TM}}$ transfection for 4 h; b, Lipofectamine-2000 ${ }^{\mathrm{TM}}$ transfection for 15 min; c-e, MNPs@PEI, MNPs@CA or MNPs@CMD based liposome-magnetofection for 15 min. A-E was the bright phase of a-e, respectively.

\subsection{Cell line-dependant magnetofection}

As the MNPs@PEI could serve as enhancers to improve the PEI-reagent transfection rate, which is also a very convenient system, we then evaluated the differences of transfection rate in different cell lines. The results showed the final transfection efficiency of magnetofection was apparently different in the three cell lines, which suggested the magne- tofection efficiency was cell line-dependant (Figure 6). In general, liposome reagent is superior to PEI reagent (used alone) owning to the high toxicity of PEI. We then experimented using MNPs as transfection enhancers to improve the transfection efficiency for PEI reagent, in comparison with the liposome reagent. PEI-magnetofection was superior to Lipofectamine- $2000^{\mathrm{TM}}$ transfection in CHO-K1 cells
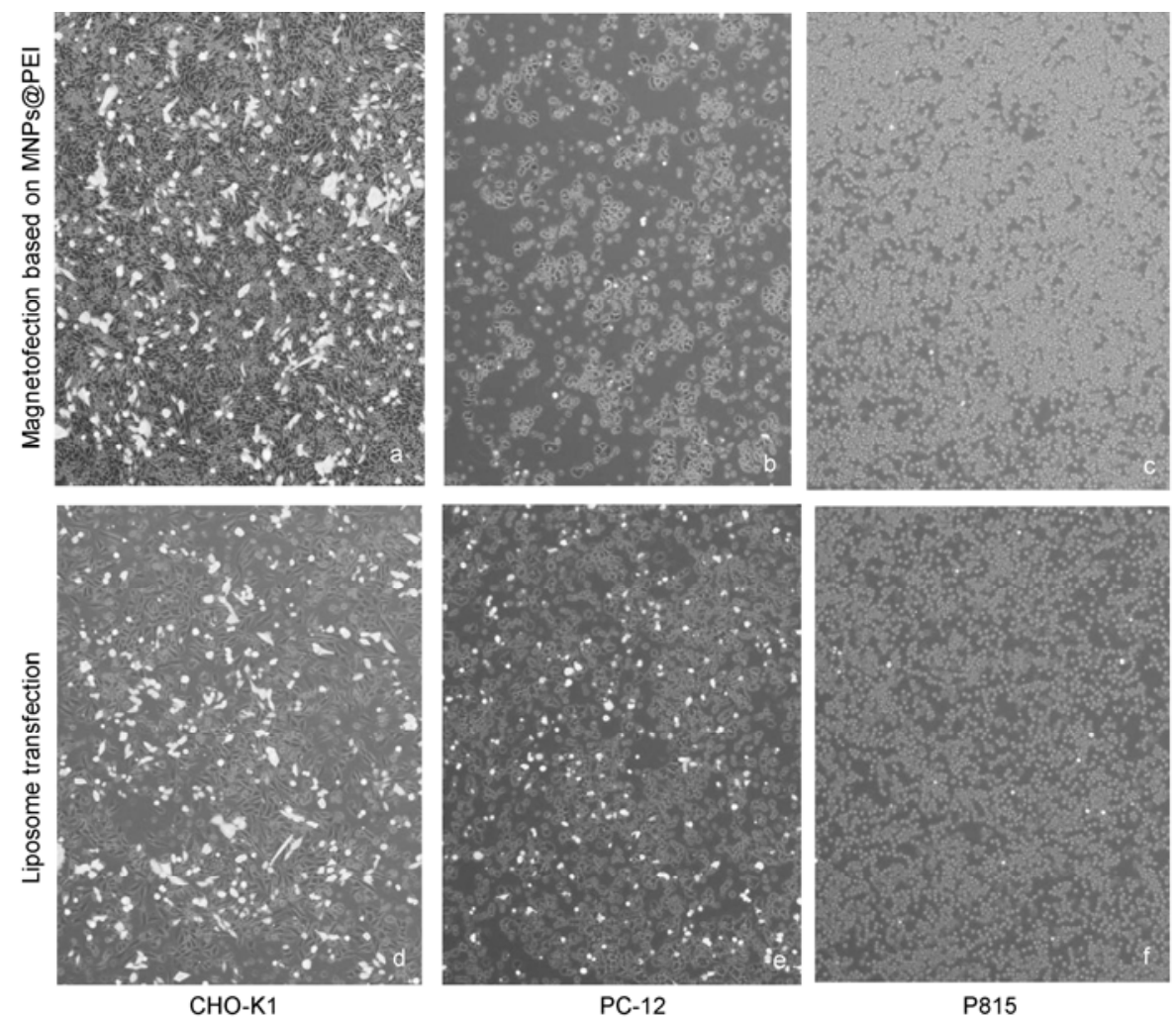

Figure 6 Using MNPs@PEI as enhancers of PEI transfection to transfect CHO-K1 cells a, PC-12 cells b and P815 cells c, respectively, and using Lipofectamine- $2000^{\mathrm{TM}}$ reagent to transfect CHO-K1 d, PC-12 e and P815 cells f, respectively. 
(Figure 6a,d), but the results were on the opposite in PC-12 cells (Figure 6b,e), and there were almost no cells transfected in the suspended cell-P815 by using either methods (Figure 6c,f). The transfection rate and the gene expression level could be improved by magnetofection, but the final transfection level achieved also depended on the original transfection reagent and the cell lines. Based on the mechanism of magnetofection in general that: (1) the magnetic field itself does not alter the uptake mechanism of magnetofectins; (2) the magnetic forces lead to an accelerated sedimentation of magnetofectins on the cell surface; and (3) do not directly affect the endocytic uptake mechanism [8], and from our results shown in Figure 6, the magnetofection has its limitation to overcome the bottle-neck problems to transfect the hard-transfected cells, such as the primary cells, the nerve cells and the suspended cells.

\section{Conclusions}

In summary, we have shown that magnetofectins are easy to be prepared by mixing charged MNPs with pDNA and vectors via electrostatic self-assembly. The as-formed magnetofectins are stable enough to meet the needs of magnetofection and apparently improve transfection rates and transgene expression level in COS-7 cells compared to standard PEI and liposome transfections. Higher transfection rates and transgene expression levels are achievable within short processing time, resulting in measurable advantages including reduction of cytotoxicity and convenience of the operation. Our transfection study in COS-7 cells would contribute to the design of magnetofection protocols. Both positively and negatively charged MNPs provide a convenient platform of making magnetofectins with comparable transfection efficiency. Taken together, we demonstrate that magnetofectins formed by self-assmebly of charged MNPs, pDNA and PEI could improve non-viral vector transfection, which could be used for targeted gene delivery in further applications.

The authors thank the Instrumental Analysis Center of Shanghai Jiao Tong University for the characterization of materials and thank Lisha Wang to provide the coated MNPs. The authors also thank Dr. Weiliang Xia for critical reading of the manuscript. This work was supported by the International Cooperation Project (20080068 and 075207012) and the National Natural Science Foundation of China (81000656).

1 Luo D, Saltzman W M. Synthetic DNA delivery systems. Nat Biotech, 2000, 18: 33-37

2 Chang Y, Bai Y P, Teng B, et al. A new drug carrier: Magnetite nanoparticles coated with amphiphilic block copolymer. Chin Sci Bull, 2009, 54: 1190-1196

3 Mintzer M, Simanek E. Nonviral vectors for gene delivery. Chem Rev, 2009, 109: 259-302

4 Huth S, Lausier J, Gersting S, et al. Insights into the mechanism of magnetofection using PEI-based magnetofectins for gene transfer. J Gene Med, 2004, 6: 923-936

5 Mykhaylyk O, Antequera Y, Vlaskou D, et al. Generation of magnetic nonviral gene transfer agents and magnetofection in vitro. Nat Prot, 2007, 2: 2391-2411

6 Pan X, Guan J, Yoo J-W, et al. Cationic lipid-coated magnetic nanoparticles associated with transferrin for gene delivery. Int $\mathrm{J}$ Pharm, 2008, 358: 8

7 Wang X, Zhou L, Ma Y, et al. Charged magnetic nanoparticles for enhancing gene transfection. IEEE Trans Nanotech, 2009, 8: 142-147

8 Jolivet J, Chanéac C, Tronc E. Iron oxide chemistry. From molecular clusters to extended solid networks. Chem Commun, 2004, 2004: 481-487

9 Kichler A, Leborgne C, Coeytaux E, et al. Polyethylenimine-mediated gene delivery: A mechanistic study. J Gene Med, 2001, 3: 135-144

10 Fischer D, Bieber T, Li Y, et al. A novel non-viral vector for DNA delivery based on low molecular weight, branched polyethylenimine: Effect of molecular weight on transfection efficiency and cytotoxicity. Pharm Res, 1999, 16: 1273-1279

11 Rémy F, Anton M, Lausier J, et al. The magnetofection method: Using magnetic force to enhance gene delivery. Biol Chem, 2003, 384: 737-747

12 Scherer F, Anton M, Schillinger U, et al. Magnetofection: Enhancing and targeting gene delivery by magnetic force in vitro and in vivo. Gene Therapy, 2002, 9: 102-109

Open Access This article is distributed under the terms of the Creative Commons Attribution License which permits any use, distribution, and reproduction in any medium, provided the original author(s) and source are credited. 